\title{
Erratum to: Histopathological Changes in Laparoscopic Sleeve Gastrectomy Specimens: Prevalence, Risk Factors, and Value of Routine Histopathologic Examination
}

\author{
Tamer Saafan $^{1}$ • Moataz Bashah ${ }^{2}$ - Walid El Ansari ${ }^{3,4,5}$ • Mohsen Karam ${ }^{2}$
}

Published online: 27 July 2017

(C) Springer Science+Business Media, LLC 2017

OBES SURG (2017) 27:1741-1749

DOI 10.1007/s11695-016-2525-1

Some author affiliations were omitted in the original article. They are presented correctly here. The name of author Tamer Saafan was misspelled and is correct here.

The online version of the original article can be found at http://dx.doi.org/ 10.1007/s11695-016-2525-1

Tamer Saafan

tsaafan@gmail.com

1 Department of General Surgery, Hamad General Hospital, Hamad Medical Corporation, Doha, State of Qatar

2 Department of Bariatric Surgery, Hamad General Hospital, Hamad Medical Corporation, Doha, State of Qatar

3 Department of Surgery, Hamad General Hospital, Hamad Medical Corporation, Doha, State of Qatar

4 College of Medicine, Doha, State of Qatar

5 School of Health and Education, University of Skövde, Skövde, Sweden 